Volume 31 (2022) 134-142

DOI: $10.24330 /$ ieja.964819

\title{
ON QUARTIC DIOPHANTINE EQUATIONS WITH TRIVIAL SOLUTIONS IN THE GAUSSIAN INTEGERS
}

\author{
Mahnaz Ahmadi and Ali S. Janfada \\ Received: 19 December 2020; Accepted: 4 July 2021 \\ Communicated by Burcu Üngör
}

\begin{abstract}
We show that the quartic Diophantine equations $a x^{4}+b y^{4}=c z^{2}$ has only trivial solution in the Gaussian integers for some particular choices of $a, b$ and $c$. Our strategy is by elliptic curves method. In fact, we exhibit two null-rank corresponding families of elliptic curves over Gaussian field. We also determine the torsion groups of both families.
\end{abstract}

Mathematics Subject Classification (2020): 11D25, 11G05, 11D45

Keywords: Diophantine equation, elliptic curves, quartic equation, number of solutions of Diophantine equations

\section{Introduction}

The integer solutions of the Diophantine equation

$$
a x^{4}+b y^{4}=c z^{2}
$$

can be mostly found in classical book on Diophantine equations. Our interest is the solutions in the Gaussian integers $\mathbb{Z}[i]$. By a trivial solution of (1) we mean $x=y=z=0$ or, $a=b=c$ and, in addition, one of the $x, y$ is zero and the square of the other equals $z$.

The Diophantine equation $x^{4}+y^{4}=z^{2}$ was studied by Fermat who proved by infinite-descent method that there exist no nontrivial solution in $\mathbb{Z}$. Hilbert [3] extended this result by showing that the equation $x^{4}+y^{4}=z^{2}$ has only trivial solution in $\mathbb{Z}[i]$. From his proof, it follows that the equation $x^{4}-y^{4}=z^{2}$ has also trivial solution in $\mathbb{Z}[i]$.

Other authors also examined similar problems. Najman [5] found all nontrivial solutions of the equations $x^{4} \pm y^{4}=i z^{2}$ in $\mathbb{Z}[i]$. He also gave a new proof of Hilbert's results. Szabó [9] solved the eight equations $x^{4}+m y^{4}=z^{2}$ in $\mathbb{Z}[i]$, where $m= \pm 2^{n}$, and $0 \leq n \leq 3$. He also considered the equations of the form (1) with only trivial solution in $\mathbb{Z}[i]$ and proved that the equations $x^{4}-p y^{4}=z^{2}$ and $x^{4}-p^{3} y^{4}=z^{2}$ have only trivial solutions in $\mathbb{Z}[i]$, where $p$ is a prime $p \equiv 3 \bmod 8$. However, the 
equations $x^{4}+p y^{4}=z^{2}$ and $x^{4}+p^{3} y^{4}=z^{2}$ have integer solutions $(1,1,2)$ and $(2,1,5)$, respectively, when, say, $p=3$.

Izadi et al. [4] studied two family of Diophantine equations of type (1) over the Gaussian integers. More precisely, they considered the families of equations $y^{4} \pm p^{3} x^{4}=z^{2}$ with $p \equiv 3 \bmod 8$ or $\bmod 16$ and $y^{4} \pm p x^{4}=z^{2}$ with $p \equiv 7$ or 11 mod 16 over the Gaussian integers and showed by elliptic curves method that in either cases there are only trivial solutions.

By a prime we shall mean a prime in $\mathbb{Z}$; we shall refer to primes in $\mathbb{Z}[i]$ as Gaussian primes. Let $p, q$ be primes $p \equiv 3 \bmod 8, q \equiv 1 \bmod 8$, and the Legendre symbol $\left(\frac{p}{q}\right) \neq 1$. In this article, we find out another equation of type (1) with only trivial solution in $\mathbb{Z}[i]$, where $a=1, b= \pm q p^{2}$ with $p, q$ as above, and $c$ is a power of $i, 1+i$, or 2 . The approach is also by elliptic curves method.

Theorem 1.1. Let $p, q$ be primes $p \equiv 3 \bmod 8, q \equiv 1 \bmod 8$, and $\left(\frac{p}{q}\right) \neq 1$. The Diophantine equations $x^{4} \pm q p^{2} y^{4}= \pm z^{2}$ and $x^{4} \pm q p^{2} y^{4}= \pm i z^{2}$ have only trivial solutions in $\mathbb{Z}[i]$.

The element $\omega=1+i$ is a Gaussian prime satisfying $\omega^{4}=-4$. Each of the Diophantine equations

$$
x^{4}+4 q p^{2} y^{4}=z^{2}, x^{4}-4 q p^{2} y^{4}=z^{2},-4 x^{4}+4 q p^{2} y^{4}=z^{2}
$$

may be transformed to one of the equations

$$
x^{4}-q p^{2} y^{4}=z^{2}, x^{4}+q p^{2} y^{4}=z^{2} .
$$

It suffices to substitute $y$ by $\omega y$ in the first two, and an extra substitution $\omega x$ for $x$ in the third. Therefore, the equations (2) also have only trivial solutions in $\mathbb{Z}[i]$. The following corollary gives even more equations of type (1) with trivial solutions.

Corollary 1.2. Let $p, q$ be primes with $p \equiv 3 \bmod 8, q \equiv 1 \bmod 8$, and $\left(\frac{p}{q}\right) \neq 1$. The Diophantine equations $x^{4} \pm q p^{2} y^{4}= \pm 2^{n} z^{2}$ and $x^{4} \pm q p^{2} y^{4}= \pm i 2^{n} z^{2}$ have only trivial solutions in $\mathbb{Z}[i]$ for any $n \in \mathbb{Z}^{+}$.

As seen, the coefficient of $z^{2}$ in each of the equations in Theorem 1.1 or Corollary 1.2 is a power of $i, 1+i$, or 2 .

Remark 1.3. Since any solution in $\mathbb{Q}(i)$ gives a solution in $\mathbb{Z}[i]$, through this work we shall consider all solutions in $\mathbb{Z}[i]$.

Elliptic curves are used to sketch the proof of Theorem 1.1. Elliptic curves over the Gaussian field $\mathbb{Q}(i)$ are not so known. We are interested the elliptic curves of 
type $Y^{2}=X^{3}+d X$ over $\mathbb{Q}(i)$. For the same family over $\mathbb{Q}$ we cite the comprehensive reference [7].

The so-called Selmer-Mordell conjecture says that the rank of elliptic curves $Y^{2}=X^{3}+p X$, with $p$ prime, over $\mathbb{Q}$ is exactly 1. Working over $\mathbb{Q}(i)$, Bremner and Cassels [1] showed that this conjecture is true for the primes $p \equiv 5 \bmod 8$ less than 1000. Other authors enlarged the upper bound but worked entirely over $\mathbb{Q}$.

Consider the two families of elliptic curves

$$
E: Y^{2}=X^{3} \pm q p^{2} X
$$

where $p, q$ are as in Theorem 1.1. We show that the Mordell-Weil rank of both families (3) over the Gaussian field $\mathbb{Q}(i)$ is zero.

Theorem 1.4. For the primes $p \equiv 3 \bmod 8$ and $q \equiv 1 \bmod 8$ with $\left(\frac{p}{q}\right) \neq 1$, the ranks of the two families of elliptic curves

$$
Y^{2}=X^{3} \pm q p^{2} X
$$

over $\mathbb{Q}(i)$ are zero.

We also determine the torsion groups of the families (3).

Theorem 1.5. For the primes $p \equiv 3 \bmod 8$ and $q \equiv 1 \bmod 8$ with $\left(\frac{p}{q}\right) \neq 1$, the torsion groups of the two families of elliptic curves

$$
Y^{2}=X^{3} \pm q p^{2} X
$$

over $\mathbb{Q}(i)$ are isomorphic to $\mathbb{Z} / 2 \mathbb{Z}$.

\section{Preliminaries}

Let $E$ be an elliptic curve over the field $\mathbb{K}$ of characteristic not equals 2 or 3 , and let $E(\mathbb{K})$ denote the $\mathbb{K}$-rational points of $E$ over $\mathbb{K}$. The so-called Mordell-Weil theorem asserts that $E(\mathbb{K})$ is a finitely generated abelian group and hence can be represented as

$$
E(\mathbb{K})=E(\mathbb{K})_{\text {tors }} \oplus \mathbb{Z}^{r}, r \geq 0,
$$

where $E(\mathbb{K})_{\text {tors }}$ denotes the torsion group of $E(\mathbb{K})$ and $r$ is called the (algebraic) rank of $E$ over $\mathbb{K}$. If $\mathbb{K}$ is quadratic field, there are 26 possible torsion groups, while in the case of the Gaussian quadratic field $\mathbb{K}=\mathbb{Q}(i)$, there are exactly 16 possible torsion groups, namely, the 15 groups from Mazur's theorem and the group $\mathbb{Z} / 4 \mathbb{Z} \times \mathbb{Z} / 4 \mathbb{Z}$ (see also [6]). To determine the torsion subgroup of $E(\mathbb{K})$ of our families (3) over $\mathbb{K}=\mathbb{Q}(i)$, we need the extended Lutz-Nagell theorem [7]. 
Theorem 2.1 (Extended Lutz-Nagell theorem). Consider the elliptic curve $Y^{2}=$ $X^{3}+a X+b$ with $a, b \in \mathbb{Z}[i]$ and let $(X, Y) \in E(\mathbb{Q}(i))$ be a torsion point. Then,

(1) $X, Y \in \mathbb{Z}[i]$;

(2) either $Y=0$ or $Y^{2} \mid 4 a^{3}+27 b^{2}$.

The plan of proving Theorems 1.4 and 1.5 is hanging on the 2-decent method for determining the rank of $E(\mathbb{Q})$. We describe briefly this method. For more details see $[2,8]$. Suppose that $E: Y^{2}=X^{3}+A X^{2}+B X$ is an elliptic curve over $\mathbb{Q}$ and $\widehat{E}: Y^{2}=X^{3}-2 A X^{2}+\left(A^{2}-4 B\right) X$ is the curve isogenous to $E$. Let $\mathbb{Q}^{*}$ be the multiplicative group of nonzero rational numbers, and $\mathbb{Q}^{* 2}$ denote the subgroup of squares of elements of $\mathbb{Q}^{*}$. Then, $\mathbb{Q}^{*} / \mathbb{Q}^{* 2}$ is the multiplicative group of rational numbers modulo squares. The process of determining the rank of $E$ requires that we look at both curves $E$ and $\widehat{E}$. Define the 2-descent homomorphism $\alpha: E(\mathbb{Q}) \longrightarrow \mathbb{Q}^{*} / \mathbb{Q}^{* 2}$ by

$$
\alpha(P)=\left\{\begin{array}{lll}
1 & \bmod \mathbb{Q}^{* 2}, & \text { if } P=\mathcal{O}, \text { the point at infinity }, \\
B & \bmod \mathbb{Q}^{* 2}, & \text { if } P=(0,0), \\
X & \bmod \mathbb{Q}^{* 2}, & \text { if } P=(X, Y) \text { with } X \neq 0
\end{array}\right.
$$

The 2-descent homomorphism $\widehat{\alpha}: \widehat{E}(\mathbb{Q}) \longrightarrow \mathbb{Q}^{*} / \mathbb{Q}^{* 2}$ is similarly defined.

$$
\widehat{\alpha}(\widehat{P})= \begin{cases}1 \quad \bmod \mathbb{Q}^{* 2}, & \text { if } \widehat{P}=\mathcal{O}, \text { the point at infinity } \\ \widehat{B} \bmod \mathbb{Q}^{* 2}, & \text { if } \widehat{P}=(0,0), \\ X \quad \bmod \mathbb{Q}^{* 2}, & \text { if } \widehat{P}=(X, Y) \text { with } X \neq 0,\end{cases}
$$

where $\widehat{B}=A^{2}-4 B$.

Proposition 2.2. With the above notations, let $r$ denote the rank of $E(\mathbb{Q})$. Then,

$$
|\alpha(E(\mathbb{Q}))||\widehat{\alpha}(\widehat{E}(\mathbb{Q}))|=2^{r+2} .
$$

A practical method to compute $|\alpha(E(\mathbb{Q}))|$ is followed by looking at the factorization of $B$. We make this more precisely in the following theorem [2].

Theorem 2.3. The group $\alpha(E(\mathbb{Q}))$ is equal to the classes modulo squares of $1, B$, and the positive and negative divisors $B_{1}$ of $B$ such that the quartic equation

$$
V^{2}=B_{1} U^{4}+A U^{2} W^{2}+\left(B / B_{1}\right) W^{4}
$$

has a solution $(U, V, W)$ with $U, V$ and $W$ pairwise coprime such that $U W \neq 0$ and

$$
\operatorname{gcd}\left(B / B_{1}, U\right)=\operatorname{gcd}\left(B_{1}, W\right)=1,
$$

and the point $P=\left(\frac{B_{1} U^{2}}{W^{2}}, \frac{B_{1} U V}{W^{3}}\right)$ is in $E(\mathbb{Q})$ such that $\alpha(P)=B_{1}$. 
In general, Theorem 2.3 gives us a method for determining the rank of $E(\mathbb{Q})$, provided that we are able to determine whether or not each of the curves generated by the divisors of $B$ and $\widehat{B}$ in the definitions of $\alpha$ and $\widehat{\alpha}$ have solutions. It is also important to note that calculating the rank of an elliptic curve using the 2-descent method can be rather time consuming, depending on how many square-free divisors of $B$ and $\widehat{B}$ there are.

The group of elliptic curves $E(\mathbb{K})$ over the quadratic fields $\mathbb{K}=\mathbb{Q}(\sqrt{m})$, with square-free integer $m$ has interesting properties. The next result [7] shows that the rank of $E$ over $\mathbb{K}$ is the sum of the ranks of $E$ and it's $m$-twist $E_{m}$ over $\mathbb{Q}$.

Theorem 2.4. Let $\mathbb{K}=\mathbb{Q}(\sqrt{m})$ be a quadratic field, where $m$ is a square-free integer. Let $E: y^{2}=x^{3}+a x^{2}+b x$ be an elliptic curve over $\mathbb{Q}$ and $E_{m}: y^{2}=$ $x^{3}+\max ^{2}+m^{2} b x$ be the $m$-twist of $E$. Then

$$
\operatorname{rank}(E(\mathbb{K}))=\operatorname{rank}(E(\mathbb{Q}))+\operatorname{rank}\left(E_{m}(\mathbb{Q})\right) .
$$

\section{Proofs}

Proof of Theorem 1.4. We prove the result for the family $E: Y^{2}=X^{3}+q p^{2} X$. The proof for the other family uses similar techniques. Appealing to Proposition 2.2 we need to prove

$$
|\alpha(E(\mathbb{Q}))||\widehat{\alpha}(\widehat{E}(\mathbb{Q}))|=4 .
$$

In other words, by the definitions of the 2-descent homomorphisms $\alpha$ and $\widehat{\alpha}$, we should prove

$$
|\alpha(E(\mathbb{Q}))|=2=|\widehat{\alpha}(\widehat{E}(\mathbb{Q}))| .
$$

We do this in two steps. Note that all calculations and equations are modulo squares.

Step 1. We show $|\alpha(E(\mathbb{Q}))|=2$. In this part, the quartic equation (4) in Theorem 2.3 is

$$
V^{2}=B_{1} U^{4}+\frac{q p^{2}}{B_{1}} W^{4}
$$

Therefore, modulo squares,

$$
B_{1} \in\{ \pm 1, \pm p, \pm q, \pm q p\} .
$$

By the definition of $\alpha, 1$ and $q$ are in the image of $\alpha$. We show that none of the $B_{1}$, except to 1 and $q$, is in $\operatorname{Im}(\alpha)$. If $B_{1}=-1$, the equation (5) turns to $V^{2}=$ $-U^{4}-q p^{2} W^{4}$, which is impossible. The case $B_{1}=-p$ has the same discussion.

For $B_{1}=p$, we get $\left(V / U^{2}\right)^{2} \equiv p \bmod q$. By the property of the Legendre symbol we get $\left(\frac{p}{q}\right)=1$, contradicts the hypothesis. 
Now, let $B_{1}=-q$. If $-q \in \operatorname{Im}(\alpha)$ then, $q \cdot(-q)=-1 \in \operatorname{Im}(\alpha)$, which is failed in the case $B_{1}=-1$. The cases $B_{1}= \pm q p$ have similar reason with different failures. Step 2. We prove $|\widehat{\alpha}(\widehat{E}(\mathbb{Q}))|=2$. Here, the quartic equation (4) in Theorem 2.3 is

$$
\widehat{V}^{2}=\widehat{B_{1}} \widehat{U}^{4}-\frac{4 q p^{2}}{\widehat{B}_{1}} \widehat{W}^{4}
$$

Therefore, modulo squares,

$$
\widehat{B_{1}} \in\{ \pm 1, \pm 2, \pm p, \pm 2 p, \pm q, \pm 2 q, \pm q p, \pm 2 q p\} .
$$

By the definition of $\widehat{\alpha}$, two elements 1 and $-q$ are in the image of $\alpha$. We show that the other elements of $\widehat{B_{1}}$ are not in $\operatorname{Im}(\widehat{\alpha})$. We distinguish some cases and try to get contradiction in each case.

3.0.1. $\widehat{B_{1}}=-1 ; \widehat{V}^{2}=-\widehat{U}^{4}+4 q p^{2} \widehat{W}^{4}$. Then, $\left(\widehat{V} / \widehat{U}^{2}\right)^{2} \equiv-1 \bmod p$. By the property of the Legendre symbol, we get $\left(\frac{-1}{p}\right)=1$, which implies $p \equiv 1 \bmod 4$, contradicts the hypothesis.

3.0.2. $\widehat{B_{1}}=2 ; \widehat{V}^{2}=2 \widehat{U}^{4}-2 q p^{2} \widehat{W}^{4}$. Hence, $\left(\widehat{V} / \widehat{U}^{2}\right)^{2} \equiv 2 \bmod p$. It follows that $\left(\frac{2}{p}\right)=1$, showing that $p \equiv 1$ or $7 \bmod 8$, contradicts again the hypothesis.

3.0.3. $\widehat{B_{1}}=p ; \widehat{V}^{2}=p \widehat{U}^{4}-4 q p \widehat{W}^{4}$. Here, we have $\left(\widehat{V} / \widehat{U}^{2}\right)^{2} \equiv p \bmod q$, which gives $\left(\frac{p}{q}\right)=1$, a contradiction.

3.0.4. $\widehat{B_{1}}=-p ; \widehat{V}^{2}=-p \widehat{U}^{4}+4 q p \widehat{W}^{4}$. Now, $\left(\widehat{V} / \widehat{U}^{2}\right)^{2} \equiv-p \bmod q$ from which, $\left(\frac{-p}{q}\right)=1$. This implies $\left(\frac{-1}{q}\right)=-1$ from which we get $q \equiv 3 \bmod 4$, which is impossible.

3.0.5. $\widehat{B_{1}}=2 p ; \widehat{V}^{2}=2 p \widehat{U}^{4}-2 q p \widehat{W}^{4}$. We obtain $\left(\widehat{V} / \widehat{U}^{2}\right)^{2} \equiv 2 p \bmod q$ and hence $\left(\frac{2 p}{q}\right)=1$. Thus, $\left(\frac{2}{q}\right)=-1$ which shows $q \equiv 3$ or $5 \bmod 8$, again impossible. The cases $\widehat{B_{1}}=2 q, 2 q p$ have similar reason.

3.0.6. $\widehat{B_{1}}=q$. If $q \in \operatorname{Im}(\widehat{\alpha})$ then, $q \cdot(-q)=-q^{2}=-1 \in \operatorname{Im}(\widehat{\alpha})$ which is failed in the case $\widehat{B_{1}}=-1$. The cases $\widehat{B_{1}}=-2,-2 q,-2 p, \pm q p,-2 q p$ are similarly failed with different failures.

We showed $\operatorname{rank}(E(\mathbb{Q}))=0$. An apply of Theorem 2.4 completes the proof of Theorem 1.4 since $m=-1$ and the $m$-twist of $E$ is itself.

Proof of Theorem 1.5. Let $(X, Y)$ be a torsion point. Then, by Theorem 2.1, $X, Y \in \mathbb{Z}[i]$ and, in addition, either $Y=0$ or $Y^{2} \mid 4 q^{3} p^{6}$. The relation $Y=0$ gives 
the 2-torsion point $(0,0)$. We claim that there is no other torsion point, that is, $Y^{2}$ never divides $4 q^{3} p^{6}$. Let by the contrary that $Y^{2} \mid 4 q^{3} p^{6}$. Then, $Y^{2}=\ell q^{s} p^{t}$, where

$$
\ell \in\{ \pm 1, \pm 2 i, \pm 4\}, s \in\{0,2\}, t \in\{0,2,4,6\} .
$$

Regardless the sign of $\ell$, we try to get contradiction in all 24 cases. The opposite sign of $\ell$ has similar discussion. We take $\omega=(1+i)$.

The case $Y^{2}=1=(-i)^{2}=X^{3}+q p^{2} X$ is clearly impossible. Let $Y^{2}=2 i=$ $\omega^{2}=X^{3}+q p^{2} X$. The only Gaussian prime factor of $X$ is $\omega$. Then, we have $\omega^{2}=\omega^{3 k}+q p^{2} \omega^{k}$ with $k \geq 1$, showing that $\cos \left(\frac{k \pi}{2}\right)=\frac{1}{2}-\frac{q p^{2}}{2^{k+1}}$, which is false. The case $Y^{2}=4=\omega^{4}$ has similar discussion.

Now, let $Y^{2}=q^{2}=X^{3}+q p^{2} X$. Then, $q \mid X$. Let $k$ be the largest power of $q$ in $X$. Then, $q^{2}=q^{3 k} X_{0}^{3}+q^{k+1} p^{2} X_{0}, k \geq 1$, where $q \nmid X_{0}$. By canceling $q^{2}$ we get an impossible equation.

For $Y^{2}=2 q^{2} i=\omega^{2} q^{2}=X^{3}+q p^{2} X$ we have again $q \mid X$. Assume that $\omega^{2} q^{2}=q^{3 k} X_{0}^{3}+q^{k+1} p^{2} X_{0}, k \geq 1$, where $q \nmid X_{0}$. Here again $k$ is taken the largest power of $q$ in $X$. Then, by canceling the $q^{2}$, we get $\omega^{2}=q^{3 k-2} X_{0}^{3}+q^{k-1} p^{2} X_{0}$. The only Gaussian prime factor of $X_{0}$ is $\omega$. Now, we get an equation in which the powers of $\omega$ in both sides do not match. The case $Y^{2}=4 q^{2}=\omega^{4} q^{2}$ has similar discussion.

Finally, let $Y^{2}=\ell q^{s} p^{t}=X^{3}+q p^{2} X$ where, as in (6), $\ell \in\{ \pm 1, \pm 2 i, \pm 4\}, s=0,2$, and $t=2,4,6$. Then, $p \mid X$. Assume $\ell q^{s} p^{t}=p^{3 k} X_{0}^{3}+q p^{k+2} X_{0}$, with $k \geq 1$ the largest power of $q$ in $X$, where $p \nmid X_{0}$. By canceling the $p^{k^{\prime}}$ with $k^{\prime}=\min \{k+2, t\}$, we obtain an impossible equation for any $\ell$ and any $s$.

We are now ready to prove the main Theorem 1.1.

Proof of Theorem 1.1. We prove the result for the equations

$$
\begin{aligned}
& x^{4} \pm q p^{2} y^{4}=z^{2}, \\
& x^{4} \pm q p^{2} y^{4}=i z^{2},
\end{aligned}
$$

with positive signs in the right hand sides. The negative cases have similar proofs. Assume that $(x, y, z)$ is a nontrivial solution of the equation (7). Dividing both sides by $y^{4}$ and putting $x / y=u$ and $z / y^{2}=v$ we get $u^{4} \pm q p^{2}=v^{2}$. Taking $X=u^{2}$, we have two equations

$$
X=u^{2}, X^{2} \pm q p^{2}=v^{2} .
$$


Now, multiplying these two equations and putting $Y=u v$ we obtain a torsion point $\neq(0,0)$ on the elliptic curves $Y^{2}=X^{3} \pm q p^{2} X$ which is impossible by Theorem 1.5 .

Now, we work on the equation (8). Here again divide both sides by $y^{4}$ and put $x / y=u$ and $z / y^{2}=v$ to get $u^{4} \pm q p^{2}=i v^{2}$. This time taking $-i X=u^{2}$, we obtain two equations

$$
-i X=u^{2},-X^{2} \pm q p^{2}=i v^{2} .
$$

By the similar manner as in the previous case, the existence of a torsion point $\neq(0,0)$ on the elliptic curves $Y^{2}=X^{3} \pm q p^{2} X$ leads to a contradiction and this completes the proof.

Proof of Corollary 1.2. In the equation $x^{4} \pm q p^{2} y^{4}= \pm 2^{n} z^{2}$ taking $n=2 k$ and $n=2 k+1$ we get, respectively, the equations

$$
x^{4}-q p^{2} y^{4}= \pm\left(2^{k} z\right)^{2}, x^{4}-q p^{2} y^{4}= \pm i\left(i \omega 2^{k} z\right)^{2},
$$

which is in the form of equations of Theorem 1.1.

In a similar way, in the equation $x^{4} \pm q p^{2} y^{4}= \pm i 2^{n} z^{2}$ taking $n=2 k$ and $n=2 k+1$ we get, respectively, the equations

$$
x^{4}-q p^{2} y^{4}= \pm i\left(2^{k} z\right)^{2}, x^{4}-q p^{2} y^{4}= \pm\left(\omega 2^{k} z\right)^{2},
$$

which is again in the form of equations of Theorem 1.1.

Acknowledgement. The authors would like to thank Alireza Abbaspour for the original idea of the issue.

\section{References}

[1] A. Bremner and J. W. S. Cassels, On the equation $Y^{2}=X\left(X^{2}+p\right)$, Math. Comp., 42(165) (1984), 257-264.

[2] H. Cohen, Number theory, Volume I: Tools and Diophantine equations, Springer, 2007.

[3] D. Hilbert, Die Theorie der algebraischen Zahlkorper, Jahresbericht der Deutschen Mathematiker-Vereinigung, 4 (1894/95), 175-535.

[4] F. Izadi, R. F. Naghdali and P. G. Brown, Some quadratic Diophantine equation in the Gaussian integers, Bull. Aust. Math. Soc., 92(2) (2015), 187-194.

[5] F. Najman, The Diophantine equation $x^{4} \pm y^{4}=i z^{2}$ in Gaussian integers, Amer. Math. Monthly, 117(7) (2010), 637-641.

[6] F. Najman, Torsion of elliptic curves over quadratic cyclotomic fields, Math. J. Okayama Univ., 53 (2011), 75-82. 
[7] J. H. Silverman, The arithmetic of elliptic curves, 2nd edition, Springer, 2009.

[8] J. H. Silverman and J. T. Tate, Rational Points on Elliptic Curves, 2nd edition, Springer, 2015.

[9] S. Szabó, Some fourth degree Diophantine equations in Gaussian integers, Integers 4, (2004), A16 (17 pp).

M. Ahmadi and A. S. Janfada (Corresponding Author)

Department of Mathematics

Faculty of Science

Urmia University

Urmia 57561-51818, Iran

e-mails: www.ahmadi5296@gmail.com (M. Ahmadi)

asjanfada@gmail.com; a.sjanfada@urmia.ac.ir (A. S. Janfada) 